\title{
An ERP Study of Autistic Traits and Emotional Recognition in Non-Clinical Adolescence
}

\author{
Ken'ichi Nixima ${ }^{1,2,3}$, Maiko Fujimori ${ }^{2,4}$, Kazuo Okanoya $^{1,2,3}$ \\ ${ }^{1}$ Department of Life Sciences, Graduate School of Arts and Sciences, the University of Tokyo, Tokyo, Japan \\ ${ }^{2}$ ERATO Okanoya Emotional Information Project, Japan Science and Technology Agency, Saitama, Japan \\ ${ }^{3}$ Emotional Information Joint Research Laboratory, Riken Brain Science Institute, Saitama, Japan \\ ${ }^{4}$ Psycho-Oncology Division, National Cancer Center Hospital East, Chiba, Japan \\ Email: cokanoya@mail.ecc.u-tokyo.ac.jp
}

Received March $16^{\text {th }}, 2013$; revised April 19 ${ }^{\text {th }}, 2013$; accepted May 17 ${ }^{\text {th }}, 2013$

Copyright (C) 2013 Ken'ichi Nixima et al. This is an open access article distributed under the Creative Commons Attribution License, which permits unrestricted use, distribution, and reproduction in any medium, provided the original work is properly cited.

\begin{abstract}
Autistic-like traits are characterized by impaired emotional recognition and proposed to be continuously distributed in the entire population. In the non-clinical population, however, the relationship between the autistic-like traits and the behavioral/physiological patterns of emotion processing ability has not been examined. In this study, we investigated the autistic traits (even including moderate-AQ participants) of typically developing adolescents, measured with the Autism Spectrum Quotient (AQ), and the spectral property of their behavioral and physiological responses during emotion-discrimination tasks. Participants were screened and divided into three groups (high-, moderate-, and low-AQ groups) according to AQ scores. Each group participated in the subsequent emotion-discrimination (using angry, happy and neutral facial expressions) experiment involving event-related potentials (ERPs). The results indicated that high-AQ group displayed significant different patterns (lower late positive potentials) of the emotional processing involved in behavioral and physiological tasks compared with the moderate-AQ group. In contrast, their behavioral and physiological patterns were comparable to those shown in the low-AQ group. Thus, in the non-clinical adolescents, the spectrum of emotion recognition patterns might show a non-linear relationship with AQ scores, even suggesting that AQ could not be considered as a simple index for emotional processing.
\end{abstract}

Keywords: Autistic Traits; AQ; Emotions; Facial Expression; Event-Related Potentials

\section{Introduction}

Autism spectrum disorders (ASD) are neurodevelopmental disorders that interrupt social functioning, language, or communication and involve stereotyped repetitive behaviors and highly restricted interests (DSM-IV; American Psychiatric Association, 1994, ICD-10; World Health Organization, 1992). Previous researches have shown that recognition of emotional facial expressions was interrupted by autism (Dawson, Webb, Carver, Panagiotides, \& McPartland, 2004; Dawson, Webb, \& McPartland, 2005; Golan, Baron-Cohen, Hill, \& Golan, 2006; Golan, Baron-Cohen, \& Golan, 2008; Wong, Fung, Chua, \& McAlonan, 2008; Wong, Fung, McAlonan, \& Chua, 2009), and deficits in emotional processing are one of the central manifestation of autism.

Autistic characteristics are proposed to be continuously distributed in the entire population and can therefore be measured in a quantitative way by using an appropriate screening tool. Baron-Cohen et al. (2001) indicated that autistic-like traits in typically developing (non-clinical) individuals are measured with the Autism Spectrum Quotient (AQ). The AQ enables quantitative investigation of autistic traits among normally developing individuals. Previous articles have used AQ to compare between individuals with high AQ score and those with low AQ score in studies of visual processing (Almeida, Dickinson, Maybery, Badcock, \& Badcock, 2010; Grinter, Maybery, Van Beek, Pellicano, Badcock, \& Badcock, 2009), mirror-neuron activity (Puzzo, Cooper, Vetter, \& Russo, 2010), or emotional processing (Poljac, Poljac, \& Wagemans, 2012). These previous studies found that the high-AQ group displayed abnormal patterns compared to the low-AQ group, which validates the assumption that autism is a continuum even in the non-clinical population.

To attain an integrated view of the autistic-like trait continuity in non-clinical population, however, we have limited knowledge of the moderate-AQ group property, because previous AQ researches just conducted the comparison between two groups (high- and low-AQ groups). Also, these studies mainly addressed cognitive or behavioral patterns, and issues involving the relationship between physiological aspects and autistic traits remained almost unaddressed.

As a step toward elucidating the emotional spectral property or linearity of autistic traits distributed in the entire population, we investigated the behavioral (measured by accuracy and reaction times), and physiological (measured by ERPs) aspects of emotional face processing in non-clinical adolescents, even including the high-, moderate-, and low-AQ groups. 


\section{Methods}

\section{Participants}

A total of 533 typical developing students (380 males, 153 females; aged $19.5 \pm 1.6$ ) at the University of Tokyo were initially screened with the Autism Spectrum Quotient-Japanese version (Wakabayashi, Tojo, Baron-Cohen, \& Wheelwright, 2004). The Autism Spectrum Quotient (Baron-Cohen et al., 2001 ) is a self-report instrument (total scores range from 0 to 50) consisting of five subscales (Social, Attention Switching, Local Detail, Communication, and Imagination). The mean score on the AQ was 22.4, and the standard deviation was 6.74. We divided participants into three groups according to AQ scores; participants with scores that were at least one standard deviation higher $(>29)$ or lower $(<16)$ than the mean were placed in the high- and low-AQ groups respectively. Participants with scores around the mean $(21-24)$ were placed in the moderate-AQ group. These three groups (high-AQ group, $\mathrm{n}=$ 97; moderate-AQ group, $\mathrm{n}=135$; low-AQ group, $\mathrm{n}=111$ ) were contacted via e-mail and invited to participate in the subsequent ERP experiment.

A total of 55 individuals (high-AQ group, $\mathrm{n}=22$; moderate-AQ group, $\mathrm{n}=17$; low-AQ group, $\mathrm{n}=16$ ) responded our invitation e-mail and agreed to participate in the ERP experiment. In the whole ERP study, 13 (out of 55) participants were excluded from the analysis due to excessive artifacts $(\mathrm{n}=11$; see EEG Recording section), equipment failure $(n=1)$, or sleepiness $(n=1)$. The high-AQ group comprised 14 adolescents (nine males and five females) aged $18-25(\mathrm{M}=20.3$, SD $=2.3)$ with $\mathrm{AQ}$ scores $>29(\mathrm{M}=31.7, \mathrm{SD}=2.4)$. The moderate-AQ group comprised 14 adolescents (nine males and five females) aged $18-22(\mathrm{M}=20.1, \mathrm{SD}=1.4)$ with AQ scores 21 - $24(\mathrm{M}=22.6, \mathrm{SD}=.9)$. The low-AQ group comprised 14 adolescents (nine males and five females) aged $18-25(\mathrm{M}=$ $20.1, \mathrm{SD}=2.0)$ with AQ scores $<16(\mathrm{M}=11.8, \mathrm{SD}=2.7)$. All participants were right handed and had no history of neurological disease. All procedures were approved in advance by the Ethics Committee of the University of Tokyo, and all participants provided written informed consent before each experiment.

\section{Stimuli}

The stimuli for this study consisted of 144 photographs of 16 healthy professional actors (targets; eight males and eight females) displaying an emotional facial expression. Targets were instructed to direct their gaze toward the lens of camera, and instructed to express three emotions (anger, happiness, and neutrality) at three levels (high, medium, and low) of intensity. Each photograph was shown to a sample of 20 healthy participants (10 males and 10 females, age range: 22 - 58; randomly sampled separate from the present ASD study) who were asked to rate the emotions experienced by targets on a 7-point Likert scale ranging from "not at all" (0) to "extremely" (6). The resulting mean ratings ranged between 0 and 5.0 (high anger: $M$ $=3.1, \mathrm{SD}=1.6$; medium anger: $\mathrm{M}=2.1, \mathrm{SD}=1.4$; low anger: $\mathrm{M}=1.7, \mathrm{SD}=1.4$; high happiness: $\mathrm{M}=4.0, \mathrm{SD}=1.4$; medium happiness: $\mathrm{M}=2.9, \mathrm{SD}=1.6$; low happiness: $\mathrm{M}=2.1, \mathrm{SD}=$ 1.3 ; neutrality: $\mathrm{M}=.2, \mathrm{SD}=.4$ ) for the 144 photographs.

\section{Procedure}

The 144 photographs were pseudo-randomized to generate a sequence of 720 trials (each photograph was shown five times). Each face was presented for $500 \mathrm{~ms}$ on a screen (15-inch monitor), and inter-stimulus intervals varied between 1500 and $2000 \mathrm{~ms}$ to eliminate the effects of any anticipation experienced by participants. Participants were asked to look at a fixation point on a monitor situated $100 \mathrm{~cm}$ in front of them and not to move their eyes. Before each block of 48 trials, participants were instructed to press one of two buttons with their thumbs that corresponded to "emotional" (angry/happy) and "neutral" expressions in response to stimuli. Presentation version 14.1 (Neurobehavioral Systems, Inc., USA) was used to present the stimuli and to record the behavioral data (reaction times and button-press responses).

\section{EEG Recording}

A 32-channel NeuroScan scan system (Neuroscan, Inc., USA) was used to record scalp EEGs ranging from nasion to inion and from the right to the left ear. Electrode impedances were kept below $10 \mathrm{k} \Omega$ before recording. The continuous signal was amplified $(\times 1000)$ and sampled at $500 \mathrm{~Hz}$ using the nasal-apex electrode as a reference. All electrodes were re-referenced to linked electrodes placed on the left and right earlobes, and a low-pass filter with a bandwidth of $30 \mathrm{~Hz}$ was applied. Vertical and horizontal electrooculograms (EOG) were also recorded to exclude trials with eye blinks and movements. ERPs and EOGs were recorded with a 100-ms pre-stimulus baseline and an 800-ms post-stimulus interval. A semiautomatic artifact-rejecttion procedure was applied to the continuous data. First, epochs containing amplitude changes exceeding $75 \mu \mathrm{V}$ for the EOG and EEG channels were excluded. Next, all epochs and channels were scanned manually for additional disturbances. ERP data including more than 480 (out of 720) noise-free trials were used for the data analysis.

\section{Data Analysis}

We analyzed the behavioral data (accuracy and reaction times) using repeated-measures ANOVA with emotion (angry/ happy/neutral) as the within-subject factor and group (high-, moderate-, and low-AQ) as the between-subjects factor. One participant in the high-AQ group was excluded from the analysis of behavioral data due to equipment failure.

The ERP data was analyzed by averaging the mean scores for each participant on the individual trials for each emotion and intensity. Using the components commonly analyzed in other ERP studies of emotional processing (Schupp, Ohman, Junghofer, Weike, Stockburger, \& Hamm, 2004; see details in discussion), the late positive potential (LPP) was identified based on the direction of the peak and the grand-average latency of each component across the groups. To identify the LPP, the average PZ amplitudes between 500 and $600 \mathrm{~ms}$ post-stimulus were analyzed using a one-way ANOVA for emotion (anger, happiness, and neutrality) and using repeated-measures ANOVA for emotion (anger, happiness) and intensity (high, medium, and low). The mean LPP amplitude of each emotion was examined with a one-way ANOVA for group (high-, moderate-, and low-AQ). We applied the Greenhouse-Geisser adjustment for the violation of the sphericity assumption and performed the least-significant-difference (LSD) post-hoc test to assess specific differences. These calculations were performed with SPSS Statistics 19 (IBM, USA). 


\section{Results}

\section{Behavioral Data}

Accuracy. The three groups did not differ significantly with respect the mean accuracy of responses $\{\mathrm{F}(2,38)=.138, p=$ n.s., power $(1-\beta$ error prob $)=.70$, effect size $\left(\eta^{2}\right)=.0070$; Figure 1(a) $\}$. Data on response accuracy reflected a significant main effect of emotion $\{\mathrm{F}(1.2,47.1)=13.98, p<.001,1-\beta$ $\left.=.98, \eta^{2}=.27\right\}$. Participants found it easier to discriminate between happy and neutral expressions than between any other pairs of expressions (anger vs neutral \& anger vs happy); this effect was beyond that for group membership $(p<.001)$.

Reaction time. We found a significant main effect of emotion on the reaction times for accurate responses $(\mathrm{F}(1.3,50.8)=$ 98.715, $p<.001,1-\beta=1.00, \eta^{2}=.72$; Figure 1(b)). The participants responded more rapidly to emotional (angry $p<.001$; happy $p<.001$ ) faces than to neutral faces. Additionally, we found a significant interaction between group and emotion $\left(\mathrm{F}(2.7,50.8)=3.843, p<.05,1-\beta=.76, \eta^{2}=.17\right)$. The post-hoc test has shown that the moderate-AQ group tended to respond more rapidly to angry or neutral expressions than did the high-AQ group, and to happy faces than did the low-AQ group $(p<.10)$.

The positivity of the average $\mathrm{Pz}$ amplitudes between 500 and $600 \mathrm{~ms}$ post-stimulus was significantly greater in response to emotional faces than to neutral faces $(\mathrm{F}(1.7,69.9)=7.637, p$ $<.01,1-\beta=.91, \eta^{2}=.16$; Figure 2(a)). Additionally, we found a significant main effect of intensity $(F(1.4,58.1)=$ $33.419, p<.001,1-\beta=1.00, \eta^{2}=.45$; Figure 2(b)) in that greater emotional intensity triggered greater positivity. Overall, this positive potential was identified as the LPP that is correlated with the emotional processing (see LPP details in discussion).

We observed a significant main effect of group beyond that of emotion (Figure 2(c); angry: $\mathrm{F}(2,39)=4.121, p<.05,1-\beta$ $=.70, \eta^{2}=.17$; happy: $\mathrm{F}(2,39)=3.322, p<.05,1-\beta=.60, \eta^{2}$ $=.15$; neutral: $\left.\mathrm{F}(2,39)=5.032, p<.05,1-\beta=.79, \eta^{2}=.21\right)$ on the mean LPP amplitudes. The moderate-AQ group showed significantly higher LPP amplitudes than did the high-AQ group in response to angry $(p<.05)$, happy $(p<.05)$, and neutral faces $(p<.01)$. The moderate-AQ group also showed significantly higher amplitudes than did the low-AQ group in response to all emotions $(p<.05)$.

\section{Discussion}

In the present study, we investigated the relationship between autistic traits and the emotion recognition to verify the emotional spectral property of autistic traits in the entire population. Our data suggest that the high-AQ (and low-AQ) group might experience greater difficulty (lower LPPs) with emotion recognition than did the moderate-AQ group.

\section{Behavioral Data}

The response accuracy of individuals in the high-AQ group was comparable to that shown by other groups on the behavioral task. This result corresponds with the Interpersonal Reactivity Index (Davis, 1980) data in our cognitive study (Nixima, data not shown) and indicates that these individuals appropriately discriminate among the emotions of others. In contrast, the high-AQ group tended to require more time to detect facial emotions, which supports the findings of a previous study (Celani, Battacchi, \& Arcidiacono, 1999). Consistent with a previous study of autistic individuals (Hubl et al., 2003), the behavioral data imply that the high-AQ group are able to correctly discriminate among emotional faces, although they sometimes require more time for processing.

It is important to note that the behavioral pattern of the low-AQ group was similar to that of the high-AQ group. They tended to require more time for emotional discrimination compared to the moderate-AQ group, but no significant differences in accuracy were observed among the three groups. The behavioral patterns in the low-AQ group should be further investigated in the future.
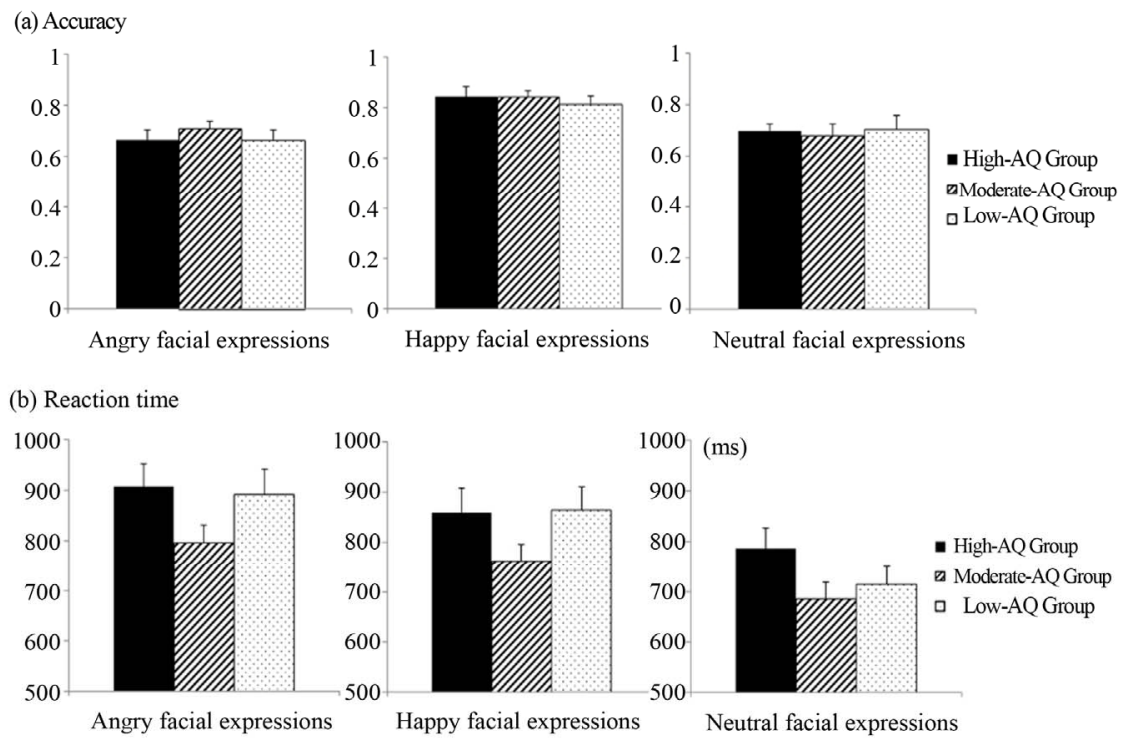

Figure 1.

(a) Accuracy results of the high-, moderate-, and low-AQ group for angry, happy, and neutral facial expressions; (b) Reaction time results of the high-, moderate-, and low-AQ group for angry, happy, and neutral facial expressions. Error bars indicate standard error of the mean (s.e.m), respectively. 


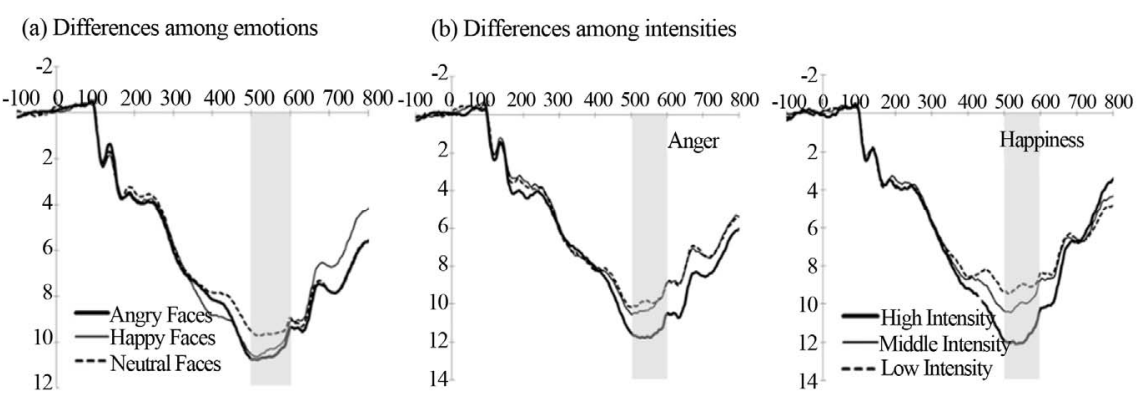

(c) Differences among AQ groups

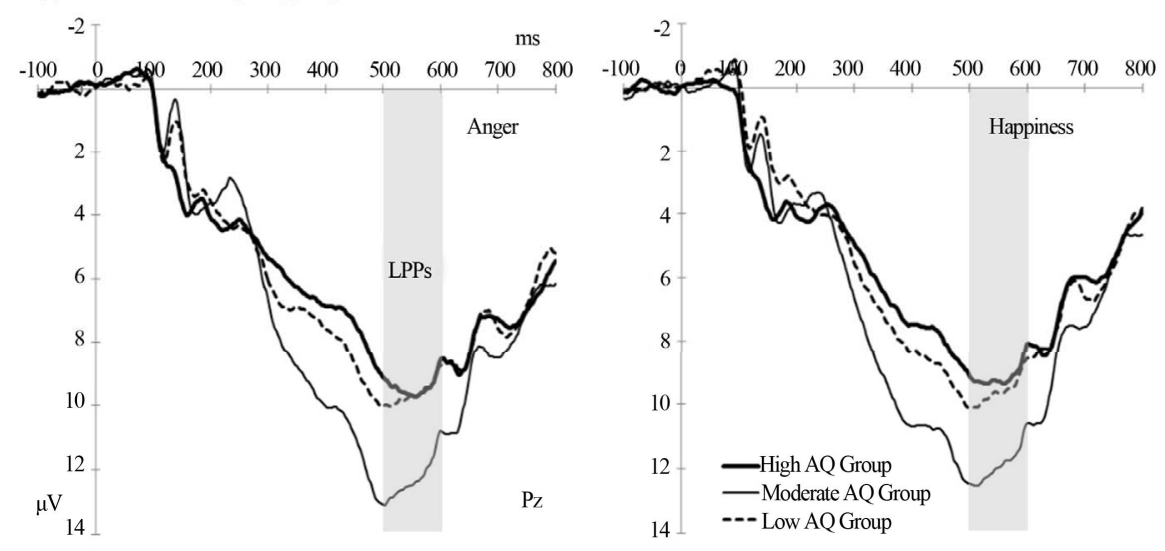

Figure 2.

(a) Grand-averaged ERPs elicited at Pz for angry, happy, and neutral facial expressions presented in a continuous sequence; (b) Grand-averaged ERPs for the high, middle, and low intensities of angry and happy facial expressions; (c) Grand-averaged ERPs for each AQ group elicited at Pz for angry and happy facial expressions.

\section{ERP Data}

We found significant group differences of late positive potentials (LPPs), late ERP components commonly analyzed in previous ERP studies of emotional processing (Schupp et al., 2004). LPPs have been considered to correlate with emotional processing and defined as the positive potentials of the ERPs over the centro-parietal sites between 400 and $600 \mathrm{~ms}$ poststimulus (Schupp et al., 2004; Schupp, Flaisch, Stockburger, \& Junghofer, 2006) that might reflect activity within neocortical brain circuits (Eimer \& Holmes, 2007). Such LPP patterns for angry and happy faces in this study were consistent with a previous ERP study (Eimer \& Holmes, 2003).

In the present study, the high-AQ group displayed significant lower LPP amplitudes than the moderate-AQ group. The neural activity difference between these two groups can be supported by the results of previous ERP studies of autistic individuals (Dawson et al., 2004; Wong et al., 2008; as described below). In contrast, the low-AQ group also demonstrated lower LPP amplitudes than did the moderate-AQ group. Although these results are consistent with the result of the reaction time patterns in this study, the similar patterns between the high- and low-AQ groups should be further investigated in the future.

The abnormal ERP patterns of autistic individuals compared with the typical developing participants have been reported by previous studies (Dawson et al., 2004; Wong et al., 2008). For example, Dawson et al. (2004) utilized high-density ERPs to investigate responses to fearful versus neutral faces in children with autism, and found that children with autism demonstrated impaired neural responses to both kinds of faces. These previ- ous researches mainly addressed early ERP components (such as P1 or N170), which might represent an early-low level stage of holistic face perception or the structural encoding of a face (Taylor, 2002; Itier \& Taylor, 2002). In our own study, we also analyzed these early ERP components at each channel, but there were no significant difference among groups (data not shown). The early ERP components (P1 or N170) are generally smaller components than LPPs, and the difference among groups in the non-clinical participants might be hardly detected.

\section{Autistic Traits and Emotional Recognition in Non-Clinical Adolescence}

The relationship of behavioral and physiological patterns between the high- and moderate-AQ group, taken together, validate the conceptualization of autistic traits as a continuum that includes normally developing individuals. We suggest that the presence of high autistic traits interrupts the instantaneous discrimination of emotions.

In contrast, the low-AQ group demonstrated comparable behavioral and physiological patterns to the high-AQ group. If this is the case, the spectrum of emotional recognition possibly shows a non-linear relationship with AQ scores. The emotional continuum of autistic traits might be ambiguous in the nonclinical population, rendering the AQ unable to identify autistic traits in those with low scores. The difference between low and moderate AQ score means little in terms of the presence of autistic traits for emotional recognition, given that the AQ, as a clinical screening tool, identifies only high autistic-like individuals. In other words, the AQ can be considered to be sensitive to 
the spectrum of emotional processing in high or moderate autistic traits, and might not adequately capture the variability in the normal range including low autistic traits. To apply the AQ for comparison with high autistic traits in normal individuals, moderate autistic traits should be more remarked than low autistic traits, from the behavioral/physiological aspect of emotional processing. Consequently, investigations of the usefulness of the AQ for quantifying autistic traits in non-clinical individuals, especially those with low autistic-like traits, must continue.

\section{Conclusion}

Our results suggest that the high-AQ group have different patterns (longer reaction times and lower LPPs) of the emotional processing underpinning behavioral and physiological responses compared to the moderate-AQ group, which affirms the autistic-like trait continuity underlying the AQ as well as the results of previous studies. This study has also shown that, similar to the high-AQ group, the low-AQ group shows a comparable pattern of emotion recognition, suggesting that the spectrum of emotion recognition in autism would show a non-linear relationship with AQ scores. The results in our study possibly mean that AQ could not be considered as a simple index for emotional processing and needs to be further investigated in the future.

\section{Acknowledgements}

This work was supported by the Okanoya Emotional Information Project of the Japan Science and Technology Agency (JST-ERATO).

\section{REFERENCES}

Almeida, R. A., Dickinson, J. E., Maybery, M. T., Badcock, J. C., \& Badcock, D. R. (2010). A new step towards understanding Embedded Figures Test performance in the autism spectrum: The radical frequency search task. Neuropsychologia, 48, 374-381. doi:10.1016/j.neuropsychologia.2009.09.024

American Psychiatric Association (2000). Diagnostic and statistical manual of mental disorders: DSM-IV-TR. Washington, DC: American Psychiatric Association.

Baron-Cohen, S., Wheelwright, S., Skinner, R., Martin, J., \& Clubley, E. (2001). The Autism-spectrum Quotient (AQ): Evidence from Asperger syndrome/high-functioning autism, males and females, scientists and mathematicians. Journal of Autism and Developmental Disorders, 31, 5-17. doi:10.1023/A:1005653411471

Celani, G., Battacchi, M. W., \& Arcidiacono, L. (1999). The understanding of the emotional meaning of facial expressions in people with autism. Journal of Autism and Developmental Disorders, 29, 57-65. doi:10.1023/A:1025970600181

Davis, M. H. (1980). A multidimensional approach to individual differences in empathy. JSAS Catalog of Selected Documents in Psychology, 10, 85 .

Dawson, G., Webb, S. J., Carver, L., Panagiotides, H., \& McPartland, J. (2004). Young children with autism show atypical brain responses to fearful versus neutral facial expressions of emotion. Developmental Science, 7, 340-359. doi:10.1111/j.1467-7687.2004.00352.x

Dawson, G., Webb, S. J., \& McPartland, J. (2005). Understanding the nature of face processing impairment in autism: Insights from be- havioral and electrophysiological studies. Developmental Neuropsychology, 27, 403-424. doi:10.1207/s15326942dn2703_6

Eimer, M., \& Holmes, A. (2007). Event-related brain potential correlates of emotional face processing. Neuropsychologia, 45, 15-31. doi:10.1016/j.neuropsychologia.2006.04.022

Eimer, M., \& Holmes, A. (2003). The role of spatial attention in the processing of facial expression: An ERP study of rapid brain responses to six basic emotions. Cognitive, Affective, \& Behavioral Neuroscience, 3, 97-110. doi:10.3758/CABN.3.2.97

Golan, O., Baron-Cohen, S., Hill, J. J., \& Golan, Y. (2006). The "reading the mind in films" task: Complex emotion recognition in adults with and without autism spectrum conditions. Social Neuroscience, 1, 111-123. doi:10.1080/17470910600980986

Golan, O., Baron-Cohen, S. \& Golan, Y. (2008). The "reading the mind in films" task [child version]: Complex emotion recognition in adults with and without autism spectrum conditions. Journal of Autism and Developmental Disorders, 38, 1534-1541. doi:10.1007/s10803-007-0533-7

Grinter, E. J., Maybery, M. T., Van Beek, P. L., Pellicano, E., Badcock, J. C., \& Badcock, D. R. (2009). Global visual processing and selfrated autistic-like traits. Journal of Autism and Developmental Disorders, 39, 1278-1290. doi:10.1007/s10803-009-0740-5

Hubl, D., Bolte, S., Feineis-Matthews, S., Lanfermann, H., Federspiel, A., Strik, W., et al. (2003). Functional imbalance of visual pathways indicates alternative face processing strategies in autism. Neurology, 61, 1232-1237. doi:10.1212/01.WNL.0000091862.22033.1A

Itier, R. J., \& Taylor, M. J. (2002). Inversion and contrast polarity reversal affect both encoding and recognition processes of unfamiliar faces: A repetition study using ERPs. NeuroImage, 15, 353-372. doi:10.1006/nimg.2001.0982

Poljac, E., Poljac, E., \& Wagemans, J. (2012). Reduced accuracy and sensitivity in the perception of emotional facial expressions in individuals with high autism spectrum traits. Autism, E-published ahead of print. doi:10.1177/1362361312455703

Puzzo, I., Cooper, N. R., Vetter, P., \& Russo, R. (2010). EEG activation differences in the pre-motor cortex and supplementary motor area between normal individuals with high and low traits of autism. Brain Research, 1342, 104-110. doi:10.1016/j.brainres.2010.04.060

Schupp, H. T., Flaisch, T., Stockburger, \& J., Junghofer, M. (2006). Emotion and attention: Event-related brain potential studies. Progress in Brain Research, 156, 31-51. doi:10.1016/S0079-6123(06)56002-9

Schupp, H. T., Ohman, A., Junghofer, M., Weike, A. I., Stockburger, J., \& Hamm, A. O. (2004). The facilitated processing of threatening faces: An ERP analysis. Emotion, 4, 189-200. doi: $10.1037 / 1528-3542.4 .2 .189$

Taylor, M. J. (2002). Non-spatial attentional effects on P1. Clinical Neurophysiology, 113, 1903-1908. doi:10.1016/S1388-2457(02)00309-7

Wakabayashi, A., Tojo, Y., Baron-Cohen, S., \& Wheelwright, S. (2004). The Autism-Spectrum Quotient (AQ) Japanese version: Evidence from high-functioning clinical group and normal adults. Shinrigaku Kenkyu Japanese Journal of Psychology, 75, 78-84. doi:10.4992/jjpsy.75.78

Wong, T. K. W., Fung, P. C. W., Chua, S. E., \& McAlonan, G. M. (2008). Abnormal spatiotemporal processing of emotional facial expressions in childhood autism: Dipole source analysis of event-related potentials. European Journal of Neuroscience, 28, 407-416. doi:10.1111/j.1460-9568.2008.06328.x

Wong, T. K. W., Fung, P. C. W., McAlonan, G. M., \& Chua, S. E. (2009). Spatiotemporal dipole source localization of face processing ERPs in adolescents: A preliminary study. Behavioral and Brain Function, 5, 16. doi:10.1186/1744-9081-5-16

World Health Organization (1992). The ICD-10 classification of mental and behavioral disorders: Clinical descriptions and diagnostic guidelines. Geneva: World Health Organization. 